\title{
COLECTORES DE ALGAS DE MÉXICO (1787-1954)
}

\author{
José Luis GodínEZ OrTEGA
}

Universidad Nacional Autónoma de México, Instituto de Biología, Apdo. postal 70-233, 04510 México, D.F. jlgo@servidor.unam.mx

\begin{abstract}
RESUMEN
Se presenta información biográfica y bibliográfica de los colectores de algas de México durante el período de 1787-1954. Los datos fueron obtenidos mediante la consulta de herbarios nacionales y extranjeros, la comunicación directa con los curadores de los mismos y la revisión de la bibliografía pertinente. Se encontraron 51 datos correspondientes a colectores, principalmente de origen norteamericano y europeo; el material colectado por los mismos fue depositado en 16 herbarios: ocho europeos, siete americanos y uno australiano. El trabajo de los colectores reúne información ficoflorística general de los phyla: Cyanobacteria, Rhodophyta, Ochrophyta (incluye Phaeophyceae y Xanthophyceae), Bacillariophyta, Chlorophyta y Charophyta; siendo los mejor representados Bacillariophyta y Rhodophyta. Se incluyen las fechas y localidades de las regiones estudiadas en 26 estados del país. En el periodo que abarca el estudio se descubrieron aproximadamente 477 nuevas especies.
\end{abstract}

Palabras clave: algas, colecciones ficológicas históricas, datos biográficos.

\begin{abstract}
Biographical as well as bibliographical information is provided about the collectors of algae in Mexico during the period 1787-1954. The data were obtained by consulting domestic and foreign herbaria, direct communication with the curators, and revision of relevant literature. Information was found concerning 51 collectors, mainly of European and American origin; material collected by them was deposited in 16 herbaria: eight in Europe, seven in America and one in Australia. The work of collectors gathers information of the phyla: Cyanobacteria, Rhodophyta, Ochrophyta (including Phaeophyceae and Xanthophyceae), Bacillariophyta, Chlorophyta and Charophyta, the best represented being Bacillariophyta and Rhodophyta. Dates and locations of the regions surveyed in 26 states are included. In the period covered by the study about 477 new species were discovered.
\end{abstract}


Key words: algae, biographical data, historical phycological collections.

\section{INTRODUCCIÓN}

En las últimas décadas los trabajos sobre algas han aumentado de manera considerable, y lógicamente también se ha incrementado el número de personas e instituciones interesadas en el campo de la ficología nacional (Ortega, 1987; Ortega y Godínez, 1994), que requieren en nuestro país de información actualizada sobre los colectores, en particular del lapso de tiempo en que estuvieron activos, de los lugares que exploraron, de los herbarios en que están depositados sus ejemplares y de los botánicos o ficólogos que estudiaron el material mexicano. Sin embargo, frecuentemente tal información no es fácil de conseguir, ya que se encuentra dispersa en herbarios principalmente extranjeros.

Se han realizado varios trabajos sobre la exploración ficológica nacional del Océano Pacífico (Pedroche et al., 2005), el Océano Atlántico (Ortega et al., 2001) y de las algas continentales de México (Ortega, 1984). Sin embargo, hasta este momento no se ha efectuado un estudio sobre los colectores de algas de México.

Según Ortega et al. (2001) y Pedroche y Sentíes Granados (2003), la exploración de las algas de México ha sido realizada en dos períodos principales. El primero con antecedentes e iniciación ficológica (1787-1954), que comprende las expediciones provenientes al principio de Europa y posteriormente también de los Estados Unidos, con el fin de conocer los recursos de flora y fauna del nuevo mundo; así como de formar herbarios de algas con colecciones de botánicos o exploradores. De esta época sobresalen W. A. Setchell y N. L. Gardner, quienes determinan y publican el material obtenido por varios colectores americanos. También se encuentra W. R. Taylor, invitado a participar en las expediciones del capitán Allan Hancock en 1939. La primera mitad del siglo XX es llamada "época Dawsoniana", en la que destaca el trabajo de E. Y. Dawson, fícólogo y colector estadounidense, cuya colección de algas es de importancia para los estudios de este grupo en el Pacífico de México. Con este investigador termina la primera época y se inicia el segundo período de la ficología formal, que será objeto de una segunda contribución, el cual comienza con las exploraciones regionales de la ficóloga mexicana Laura Huerta Múzquiz, de la Escuela Nacional de Ciencias Biológicas del Instituto Politécnico Nacional, cuyas primeras colectas fueron en mayo de 1955 en la Sonda de Campeche, Isla Pérez, Isla Cozumel e Isla Mujeres (Ortega et al., 2001). Es en este momento cuando se abre una nueva época de la ficología nacional, que conlleva la recolección de algas 
por colectores principalmente mexicanos y la creación de importantes herbarios en México (Ortega y Godínez, 1994).

La historia de los colectores de algas de México está relacionada desde la época de la colonia con el desarrollo de la botánica de plantas vasculares en el país, a partir del influjo ordenador de Linneo con el respaldo de un herbario organizado, de la llegada de la Real Expedición Científica a Nueva España y de la primera cátedra de botánica, que incluyeron las algas en sus clases y muestreos (Herrera et al., 1998). En sus inicios el avance fue lento, debido en parte a la dificultad de explorar el ambiente acuático y de los métodos de herborización para el material húmedo. No obstante tales dificultades fueron vencidas y en la actualidad existen materiales bien conservados de los siglos XVIII y XIX. Durante esta época se realizaron grandes expediciones enviadas por gobiernos o instituciones científicas de Europa y Estados Unidos de América, muchas de las cuales se ocuparon también de buscar representantes de diversos grupos de criptógamas.

Los colectores de algas no necesariamente fueron los que se dedicaban a la ficología, algunos eran estudiosos de plantas vasculares, zoólogos o comerciantes, que enviaron sus ejemplares a los especialistas de la época. En general los materiales obtenidos no fueron muy numerosos si los comparamos con lo que rindieron los grandes colectores botánicos. Así, Pringle distribuyó aproximadamente 10,000 números de plantas vasculares y solamente cerca de un centenar de algas de México. Liebmann y Schott enviaron sus ejemplares a los ficólogos J. Agardh y W. H. Harvey en siglo XIX, mientras que Carl August Ehrenberg muestreó microalgas para las investigaciones de su hermano Christian Gottfried, realizadas en Alemania. En el siglo XX, Schmitt, Howell, Mason, Johnston, Taylor y Dawson fueron los exploradores con las mayores colectas de algas de México y su material fue estudiado por destacados especialistas como W. A. Setchell y los mismos W. R. Taylor y E. Y. Dawson, entre otros.

El presente trabajo principalmente integra a los colectores que tienen colecciones (exsiccata) de algas marinas y dulceacuícolas depositadas en herbarios institucionales nacionales y extranjeros; sin menoscabo de aquellos cuyas colectas fueron incidentales o accesorias, pero que en su conjunto forman un acervo valioso. El objetivo de este estudio es presentar información sobre los colectores de algas (1787-1954) con datos biográficos y bibliográficos, las localidades de exploración, las fechas de recolección y los herbarios en donde se encuentran sus ejemplares, al mismo tiempo que constituye un reconocimiento a su labor científica; todo esto con la finalidad de apoyar el trabajo fico-florístico y taxonómico de las algas en México. 


\section{MÉTODOS}

La relación de colectores fue obtenida a través de la consulta de algunos herbarios nacionales y extranjeros más importantes (Cuadro 1); además de la revisión bibliográfica pertinente (Dawson, 1944; Ortega, 1984; Ortega et al., 2001; Pedroche y Sentíes Granados, 2003). Asimismo algunos datos fueron obtenidos mediante la consulta del Index Herbariorum (Holmgren y Holmgren, 1998) o directamente por correspondencia con los curadores de los herbarios de México, Estados Unidos, Alemania y Australia. La Escuela Nacional de Ciencias Biológicas custodia uno de las colecciones ficológicas más importantes de México (ENCB); sin embargo sus materiales son posteriores a 1954. La información biográfica fue tomada de las obras generales de Hemsley (1891), Lanjouw y Stafleu (1954, 1957), Barnhart (1965), Chaudhri et al. (1972), Vegter (1976, 1983), Stafleu y Cowan (1976, 1979, 1981, 1983, 1985, 1986) y Anónimo (1986).

El formato utilizado para cada entrada comienza con el nombre completo, fechas de nacimiento y muerte, profesión o trabajo, nacionalidad, datos relacionados con el colector, una guía somera sobre los taxa trabajados, las localidades visitadas y las fechas correspondientes, los herbarios en donde se encuentran depositados sus ejemplares de algas procedentes de México, referencias con datos biográficos y de identificación del material y finalmente información sobre los nombres de algunas de las especies que le fueron dedicadas. Sin embargo este formato no se aplicó estrictamente en todos los casos, ya que dependió de la información disponible.

\section{RESULTADOS}

ARSÈNE, Gustave Joseph Brouard (1867-1938). Educador religioso francés de las escuelas Lasallistas, llega a México en 1906 y es ubicado en el colegio Lasallista de Puebla en donde inicia su labor botánica. Tiene comunicación con ficólogos de Instituto de Botánica en Montpellier, Francia (Purata Velarde, 1981). Sus especímenes de algas Charophyta son de Puebla, fueron obtenidos en 1907 y se encuentran resguardos en BM, MEXU y US.

BARKLEY, Fred Alexander (1908-1989). Botánico norteamericano. Tiene colectas incidentales de cianobacterias en Río Santa Catarina, Nuevo León de 1944; un ejemplar se encuentra depositado en F. 
Cuadro 1. Herbarios institucionales con colecciones de algas de México. Las siglas de los herbarios y colecciones se presentan de acuerdo con Holmgren et al. (1990).

\begin{tabular}{|c|c|c|}
\hline Siglas* & Ciudad & Institución \\
\hline BHU & Berlin & $\begin{array}{l}\text { Herbarium, Bereich Botanik und Arboretum, Museum für } \\
\text { Naturkunde der Humboldt-Universität (Ehrenberg Collection) } \\
\text { Humboldt-Universität zu Berlin }\end{array}$ \\
\hline $\mathrm{BM}$ & Londres & Herbarium, Botany Department, The Natural History Museum \\
\hline $\mathrm{C}$ & Copenhague & Herbarium, Botanical Museum, University of Copenhagen \\
\hline $\mathrm{F}$ & Chicago & $\begin{array}{l}\text { Herbarium, Botany Department, Field Museum of Natural } \\
\text { History }\end{array}$ \\
\hline $\mathrm{L}$ & Leiden & $\begin{array}{l}\text { Rijksherbarium Nationaal Herbarium Nederland, Leiden } \\
\text { University Branch }\end{array}$ \\
\hline LD & Lund & Herbarium, Botanical Museum \\
\hline MA & Madrid & Real Jardín Botánico de Madrid \\
\hline MEL & Victoria & National Herbarium of Victoria, Royal Botanic Gardens \\
\hline MEXU & México, D.F. & $\begin{array}{l}\text { Herbario Nacional, Instituto de Biología, Universidad Nacional } \\
\text { Autónoma de México }\end{array}$ \\
\hline $\mathrm{MICH}$ & Ann Arbor & Herbarium, University of Michigan \\
\hline NY & Nueva York & Herbarium, New York Botanical Garden \\
\hline PC & París & $\begin{array}{l}\text { Herbier, Laboratoire de Cryptogamie, Muséum National } \\
\text { d'Histoire Naturelle }\end{array}$ \\
\hline $\mathrm{PH}$ & Philadelphia & $\begin{array}{l}\text { Herbarium, Botany Department, Academy of Natural } \\
\text { Sciences of Philadelphia }\end{array}$ \\
\hline $\mathrm{S}$ & Estocolmo & $\begin{array}{l}\text { Herbarium, Botany Departments, Swedish Museum of Natural } \\
\text { History }\end{array}$ \\
\hline $\mathrm{UC}$ & Berkeley & University Herbarium, University of California \\
\hline US & $\begin{array}{l}\text { Washington, } \\
\text { D.C. }\end{array}$ & $\begin{array}{l}\text { United States National Herbarium, Botany Department, } \\
\text { Smithsonian Institution (Francis Drouet \& Dawson Collection) }\end{array}$ \\
\hline
\end{tabular}

BRANDEGEE, Townshend Stith (1843-1925). Ingeniero y botánico norteamericano establecido en California y asociado al Departamento de Botánica en la Universidad de California. Brandegee junto con W. E. Bryant, en la tercera expedición de la Academia de Ciencias de California, obtuvieron un considerable número de ejemplares marinos, principalmente de Sargassum (Ochrophyta) de Baja California Sur en 1890 (Dawson, 1944). También colectó algunas Charophyta de Baja 
California y Baja California Sur en 1883. Sus ejemplares se encuentran depositados en NY y UC. Su material fue estudiado por Setchell y Gardner (1924). Le fueron dedicados los nombres de algunas especies de algas (p.e. Sargassum brandegeei Setchell \& N. L. Gardner, Codium brandegeei Setchell \& N. L. Gardner).

BRYANT, Walter E. (1861-1905). Ornitólogo y curador de la Academia de Ciencias de California (Fisher, 1905). Véase Brandegee.

CARLSON, Margery Claire (1892-1985). Botánica norteamericana de Northwestern University y del Field Museum of Natural History, Chicago. Fue de las mujeres pioneras de la ciencia y la conservación en Estados Unidos. Se desempeñó como colectora de plantas de México y América Central en los años cuarenta y principios de la década de los cincuenta. Obtuvo algunos especímenes de cianobacterias en Comitán, Chiapas y en Guanajuato en 1949; sus ejemplares se encuentran en F y PH.

CLARK, H. W. Colector posiblemente norteamericano, del cual se tiene poca información. Realizó una colecta incidental en Isla Guadalupe (Baja California) en 1932; su material se encuentra en UC.

DAWSON, Elmer Yale (1918-1966). Botánico, ficólogo e incansable colector norteamericano de algas marinas. Participó en la Allan Hanckock Pacific Expedición al Golfo de California a bordo del Velero III (1940) y IV (1949, 1954) (Garth, 1967). Hizo también expediciones al Pacífico mexicano bajo los auspicios de la Fundación A. Guggenheim (1946-1947) y en 1953 fue integrante de la Expedición a la Isla San Benedicto. Tuvo diferentes cargos después de pertenecer a la Allan Hanckock Foundation hasta llegar a ser el curador de botánica criptogámica del Instituto Smithsoniano. Además de ficólogo fue colector de cactáceas, grupo en el que describió muchas especies nuevas (Hawkes, 2005). Fue discípulo y amigo de William A. Setchell, prominente ficólogo del siglo XX. Dio a conocer 164 nuevos taxa de Chlorophyta, Phaeophyceae y Rhodophyta. En el catálogo de las algas marinas bentónicas del Pacífico mexicano se citan 5000 registros de especímenes de Dawson (Pedroche y Sentíes Granados, 2003). Colectó principalmente en el Pacífico de México, incluyendo Baja California, Baja California Sur, Islas Revillagigedo, el Golfo de California, Sonora y Chiapas. Una lista de las localidades visitadas y estudiadas puede consultarse en Dawson (1953). En el último trabajo que publicó sobre algas de México llegó hasta el número de colecta 27,570 correspondiente a Puerto Peñasco, Sonora 
en 1966 (Dawson, 1966). También recibió de varias personas colectas de Baja California (W. Williams, E. Flyn, C. L. Hubbs y B. Osorio Tafall en 1946; K. Norris en 1952 y M. Neushull en 1957). En un período de 26 años (1940-1966) recolectó 40,000 ejemplares, los que se encuentran en UC, US (Hawkes, 2005) y en (C). En US están registrados 65 tipos suyos de algas de México. Publicó 112 trabajos de interés ficológico, de los cuales 54 están relacionados con las macroalgas de nuestro país, 52 sobre cactáceas y otros temas (Silva, 1967). Su obra cumbre fue Marine red algae of Pacific Mexico (1953-1963). Las listas de sus publicaciones pueden consultarse en Abbott (1966), Silva (1967), Garth (1967), Pedroche et al. (2003) y Hawkes (2005). Le han dedicado los nombres de 17 taxa de algas marinas (p.e. Rhodymenia dawsonii Taylor, Codium dawsonii Silva).

DÍAZ, A. Explorador probablemente mexicano del cual se sabe muy poco. Incidentalmente colectó Codium isthmocladum Vickers (Chlorophyta) y Eucheuma isiforme (C. Agardh) J. Agardh (Rhodophyta) de Sisal, Yucatán en 1864; sus ejemplares se encuentran en $\mathrm{F}$.

DIGUET, Léon (1859-1927). Etnólogo francés originario de Havre. Estuvo encargado de la exploración científica en México entre 1893 y 1913 (Anónimo, 1986). Su colección consistió principalmente de coralináceas (Lithothamnion margaritae Hariot, Rhodophyta) de Bahía de la Paz, Baja California Sur y dos cianobacterias de Mulegé, Baja California Sur y Ensenada, Baja California, en 1894. Sus colectas se encuentran resguardadas en el herbario de París (PC). Las algas que recolectó fueron estudiadas y los resultados publicados por Paul Harriot en Algues du Golfe de Californie, recueillies par M. (Monsieur) Diguet (Harriot, 1895); posteriormente fueron reexaminadas por M. Foslie, F. Heydrich y P. Lemoine. Le fue dedicado el nombre Lithothamnion diguetii Hariot (actualmente Lithophyllum diguetii (Hariot) Heydrich).

DROUET, Francis (1907-1982). Incansable ficólogo norteamericano que examinó y reestudió 100,000 especímenes de cianobacterias (Forest, 1983). En México, junto con D. Richards, L. D. Alvarado y W. A. Lockhart, recolectó algas azul-verdes de Sonora en 1939. Su amplia colección se encuentra actualmente en US y otro tanto en F.

EHRENBERG, Carl August (1801-1849). Comerciante alemán y colector de plantas, hermano del famoso ficólogo Christian Gottfried Ehrenberg; los hermanos 
Ehrenberg se encuentran entre los más renombrados investigadores y colectores de algas microscópicas en tierras de Norteamérica, México y Sud-América, así como de las Antillas (Ehrenberg C. G., 1841(1843), 1854, 1874). En México Carl August recolectó numerosas diatomeas (Bacillariophyta) de Coahuila, Jalisco, Michoacán, Guanajuato e Hidalgo (Real del Monte) entre 1831 y 1840. Estas muestras se encuentran en una colección especial en el Museum für Naturkunde de la Universidad Humboldt en Berlín (BHU), la cual está en proceso de recatalogación. Muchas de ellas son consideradas tipo de especies de amplia destribución mundial. La historia y el manejo de esta colección pueden consultarse en Lazarus y Jahn (1998). A los Ehrenberg les han dedicado los nombres de 179 taxa de microalgas.

FASSETT, Norman Carter (1900-1954). Botánico norteamericano de Madison y colector incidental de algas Charophyta en la Laguna de Zempoala y Nuevo León en 1950; los ejemplares correspondientes se encuentran en MEXU.

FOUT, J. (siglo XIX). Colector probablemente norteamericano del que se sabe poco; incidentalmente colectó ejemplares de Bryothamnion triquetrum (Gmelin) Howe (Rhodophyta) de Celestún, Yucatán; los ejemplares se encuentran resguardados en $\mathrm{F}$, sin fecha de colecta.

GARDNER, Nathaniel Lyon (1864-1937). Notable ficólogo norteamericano, profesor de la Universidad de California. Desde 1903 fue colaborador del destacado ficólogo W. A. Setchell (1864-1943) de la misma institución. Sus abundantes colecciones son principalmente del Pacífico de Norteamérica. Setchell colectó en California únicamente, pero Gardner se aventuró desde Alaska hasta la península de Baja California, de donde describió una nueva especie: Gigartina serrata N. L. Gardner (sinónimo de Chondracanthus serratus (N. L. Gardner) J. R. Hughey \& Hommersand). Obtuvo también muestras en Todos Santos, Baja California Sur (Gardner, 1927; Setchell, 1937). Su amplia colección se encuentra en UC.

GAUMER, George Franklin (1850-1929). Médico y naturalista norteamericano que residió en Yucatán desde 1884 hasta su muerte. Realizó alrededor de 25,000 colectas de plantas vasculares entre los años 1895 y 1921 (Campos-Ríos y Chiang Cabrera, 2006). En cuanto a algas obtuvo ejemplares de Chara hornemannii Wallman de Yucatán (Charophyta), así como especímenes marinos de Sargassum filipendula C. Agardh (Ochrophyta), Bryothamnion seaforthii (Turner) Kützing, B. triquetrum (Gmelin) Howe (Rhodophyta) y también algunas cianobacterias de Yu- 
catán y Quintana Roo. Hacía envíos a Paul C. Standley. Sus colecciones se encuentran en BM, F, L, NY, PH y US (Drouet Collection).

GENTRY, Howard Scott (1903-1993). Botánico norteamericano dedicado al estudio de los agaves, que ocasionalmente recogió un ejemplar de Charophyta en 1950; el espécimen se encuentra en MEXU.

HAHN, Ludwig (1836-1881). Horticultor alemán que se adhirió a la Comisión Científica Francesa en México. Recogió algas del género Oedogonium (Chlorophyta) de México entre 1865 y 1866. Sus colecciones se encuentran en Estocolmo (S). Las algas fueron descritas por los botánicos suecos V. B. Wittrock y C. F. O. Nordsted y las incluyeron en su colección Algae aquae dulcis exsiccatae (Hirn, 1900).

HOWELL, John Thomas (1903-1994). Botánico norteamericano y asistente de la Dra. A. Eastwood; posteriormente curador de botánica de la Academia de Ciencias de California, dedicado principalmente a las fanerógamas y ocasionalmente activo como colector de algas. Participante en la Templeton Crocker Expedition de la Academia de Ciencias de California que abarcó desde Isla Guadalupe hasta las Islas Galápagos. Obtuvo material ficológico (macroalgas marinas) de la Península de Baja California especialmente en San Bartolo, Bahía San Bartolomé, Bahía Santa María, Isla San Martín, San José del Cabo, Bahía San Lucas, Bahía Tortuga e Isla Natividad, en 1932. También en las Islas Revillagigedo (Colima), Bahía Banderas (Jalisco) y Manzanillo (Sinaloa). Sus ejemplares se encuentran en UC y fueron estudiados por W. A. Setchell, quien registra 250 especies colectadas por Howell (Setchell y Gardner, 1937). Setchell le dedicó los nombres de varias especies (p.e. Sargassum howellii Setchell y Weeksia howellii Setchell \& N. L. Gardner).

HUMBOLDT, Alexander von (1769-1859). Científico alemán y explorador en la América tropical. Durante su estancia en España, en Madrid consiguió una audiencia con Carlos IV y la reina María Luisa de Parma. Conquistó la confianza de la familia real y después de los trámites correspondientes, el gobierno le hizo extender un pasaporte especial por el Consejo de Indias en el que Humboldt designaba a Aimé Bonpland como su amigo y secretario. Asimismo indicaba con la mayor precisión posible el itinerario del viaje (Beck, 1971). En 1804, cuando su expedición partió de Veracruz rumbo a La Habana a bordo de la fragata española La O, recolectaron el alga Fucus vesiculosus Linnaeus a la deriva en el Golfo de México (Bonpland et al., 1825); los mismos Humboldt y Bonpland señalaron en la etiqueta 
del ejemplar de herbario (Herb. Agardh 364, LD) lo siguiente: "Crescit in sinu mexicano, inter Veracruz et Habana. An e longinquo allata specimina?" (Crece en el Golfo de México, entre Veracruz y La Habana. ¿Probablemente especímenes traídos de un lugar lejano?). Dicha alga fue enviada a Carl Adolf Agardh, profesor de Botánica en la Universidad de Lund (Suecia) y prominente ficólogo quien la identificó y citó entre otras algas americanas en la obra de Carl Sigismund Kunth (Agardh C., 1822), diligente colaborador de Humboldt. En la actualidad la presencia de Fucus en aguas tropicales es improbable, ya que su distribución conocida se restringe a aguas frías del Atlántico Norte.

JOHNSTON, Ivan Murray (1898-1960). Botánico norteamericano y participante de la Expedición al Golfo de California patrocinada por la Academia de Ciencias de California en 1921. Estudiante graduado de Berkeley, que posteriormente formó parte del personal del Arnold Arboretum de la Universidad de Harvard (Papenfuss, 1976). Colector de algas marinas de los phyla Cyanobacteria, Rhodophyta, Ochrophyta y Chlorophyta en Baja California, en particular en Isla San Lorenzo, Isla San Pedro Mártir y en el Golfo de California en Bahía San Francisquito, Isla Ángel de la Guarda, Isla Pond, Bahía Coyote, Isla Partida, Isla San Marcos e Isla Smith, en Sonora Isla San Esteban e Isla San Jorge en 1921. También obtuvo materiales en la Península de Baja California en 1932, esta vez en Isla Cedros, Bahía San Bartolo, Bahía Santa María, San José del Cabo, Isla Natividad, Bahía San Lucas e Islas Revillagigedo. Sus ejemplares se encuentran en UC, aunque en US está depositado un isotipo de Eucheuma uncinatum Setchell \& N. L. Gardner (Rhodophyta) de Isla San Lorenzo, Baja California. Le fueron dedicados los nombres de Sargassum jonnstonii Setchell \& N. L. Gardner, Gelidium johnstonii Setchell \& N. L. Gardner y Polysiphonia johnstonii Setchell \& N. L. Gardner.

KIENER, Walter (1894-1959). Personaje norteamericano del cual poco se conoce. Recolectó algunas cianobacterias en Barranca de Oblatos y río Primavera en Jalisco en 1944. Sus ejemplares se encuentran en F y US.

LEIBOLD, Friedrich Ernst (1804-1864). Jardinero y explorador botánico alemán, que viajó a México y Cuba entre 1839 y 1844 (Stafleu y Cowan, 1979). Su material ficológico (Rhodophyta), sin localidad específica, fue entregado a Liebmann y sus especímenes se encuentran en el herbario de Copenhague (C), en el de Leiden (L) y en la colección Sonder en Australia (MEL). Otto W. Sonder fue un farmacéutico de Hamburgo que realizó una extensa colección de algas marinas de 
Australia (1812-1881), por muchos años resguardada en el herbario N. Binder en Hamburgo, y que posteriormente fue adquirida por el Herbario Nacional de Victoria, Australia (Ducker, 1981). Sonder nunca visitó México, pero recibió algunos ejemplares procedentes del país, obtenidos por Leibold y Liebmann e identificados por su amigo Kützing, ficólogo alemán. (Véanse las entradas de Liebmann y Müller).

LIEBMANN, Frederik Michael (1813-1856). Explorador botánico de Elsenöre, Dinamarca. Con el apoyo del Rey de Dinamarca, Federico VII, emprendió entre 1841 y 1843 un viaje de exploración botánica por México. Cuando regresó a su país fue profesor y posteriormente (1852) fue nombrado director del Jardín Botánico de Copenhague (Christensen, 1924-1926). Además de plantas vasculares, también recolectó macroalgas marinas y dulceacuícolas de Veracruz (Febrero de 1841), Banco de Campeche y Oaxaca (Octubre de 1842) (Liebmann, 1846). Su colección más importante se encuentra en el Museo Botánico de la Universidad de Copenhague (C), en la Universidad de Lund (LD), en el Riksherbarium de Leiden (L), en Estocolmo (S) y en Australia (MEL). Liebmann (1846) envió sus ejemplares para identificación a Jacob Georg Agardh, ficólogo sueco, hijo de Carl Adolph Agardh (Agardh, J., 1847, 1848). Algunos de los sitios visitados por este colector del lado del Océano Pacífico (San Agustín y Pochutla, Oaxaca, en octubre de 1842) y en el litoral de Veracruz (Santa María Alpatlahuac, en septiembre de 1841), resultaron ser localidades tipo de varias algas nuevas. La información acerca de su itinerario en México y de sus publicaciones puede consultarse en Liebmann (1869), Christensen (1924-1926), Ortega (1984), McVaugh (1987) y Ortega et al. (2001).

LINDEN, Jean Jules (1817-1898). Horticultor de Luxemburgo y explorador botánico de plantas vasculares que vino a México entre 1834 y 1840, bajo el auspicio belga. Incidentalmente colectó en Campeche algunos ejemplares de algas marinas (Eucheuma isiforme var. denudatum D. P. Cheney, Bryothamnion seaforthii (Turner) Kützing, Gracilaria cervicornis (Turner) J. Agardh (Rhodophyta)); los especímenes se encuentran en BM.

LLOYD, Francis Ernest (1868-1947). Fisiólogo vegetal británico y explorador en México. Su principal trabajo se enfocó sobre las plantas vasculares, aunque colectó un ejemplar de Nostoc commune var. flagelliforme (Berkeley \& Curtis) Bornet $\&$ Flahault (Cyanobacteria) de Mazapil, Zacatecas, el cual se encuentra en la Phycoteca Boreali Americana de MEXU. 
LUNDELL, Cyrus Longworth (1907-1994). Botánico norteamericano y explorador que, junto con su esposa Amelia Lundell, se interesaron en la cultura maya y las plantas vasculares de la Península de Yucatán (Campos-Ríos y Chiang Cabrera, 2006). Colectó en Quintana Roo un ejemplar de cianobacteria en el Lago Zotz, en 1933, actualmente desaparecido; el espécimen se encuentra en PH.

MACDOUGAL, Daniel Trembly (1865-1958). Botánico norteamericano, director del Carnegie Desert Laboratory en Tucson, Arizona entre 1905 y 1928. Aunque interesado mayormente en las plantas vasculares, fue el primero en colectar macroalgas marinas en el norte del Golfo de California (Bahía San Felipe) en 1904 (Dawson, 1944). Sus ejemplares se encuentran en PC, entre otros herbarios; fueron estudiados por Howe (1911). Dawson le dedicó los nombres de algunas especies (Gigartina macdougalii Dawson, Sargassum macdougalii Dawson y Codium macdougalii Dawson).

MALDONADO KOERDELL, Manuel (1908-1973). Paleontólogo y zoólogo mexicano que incidentalmente colectó un espécimen de cianobacteria en El Nogalar, norte de Monterrey, Nuevo León, en 1944; los ejemplares se encuentran en F y PH.

MARCHANT, Dr. Colector norteamericano del cual se desconoce mayor información, pero junto con su esposa Daisy R. Marchant colectaron algas de los taxa Chlorophyta, Phaeophyceae y Rhodophyta en 1917 en varias localidades de Baja California Sur (La Paz, Eureka, Santa Rosalía), Sinaloa (Mazatlán) y Sonora (Guaymas). Sus ejemplares se encuentran principalmente en PC, ocho isotipos de México en US y otros en UC. Se les dedicaron los nombres de Hypnea marchantae Setchell \& N. L. Gardner y Polysiphonia marchantae Setchell \& N. L. Gardner.

MASON, Herbert Louis (1896-1994). Botánico norteamericano y estudioso de la fitogeografía. Participante en la expedición a las Islas Revillagigedo patrocinada por la Academia de Ciencias de California. Llegó a ser profesor emérito de botánica en la Universidad de California en Berkeley. Colectó extenso material ficológico marino en Isla Guadalupe e Islas Revillagigedo en 1925. Sus ejemplares fueron estudiados y los resultados publicados por Setchell y Gardner (1930). Sus colectas se encuentran en UC y es honrado con el nombre del género Masonophycus, así como con los de varias especies (Papenfuss, 1976). 
MOCIÑO, José Mariano (1757-1820). Médico y naturalista novohispano originario de Temascaltepec. Fue integrante de la Real Expedición a Nueva España bajo las órdenes de Martín de Sessé y La Casta. Su interés principal versó sobre la flora (vascular) de México. Del material ficológico colectado por Mociño, actualmente se conservan en el Herbario Real Jardín Botánico de Madrid (MA) cuatro ejemplares que indican en la etiqueta únicamente Plantae Novae Hispaniae (1787-1795-1804) de Sessé, Mociño, Castillo y Maldonado; estos fueron determinados por P. Ponce de León en 1964 y corresponden a: Tricleocarpa cylindrica (Ellis \& Solander) Huisman \& Borowitska (MA 3911), Jania adhaerens Lamouroux (MA 3914), Dichotomaria marginata (Ellis \& Solander) Lamarck (MA 3892) y D. obtusata (Ellis \& Solander) Lamarck (MA 3912). Dichas algas se distribuyen en la región tropical del Atlántico occidental. Del material de agua dulce de esta expedición se conservan algas de los géneros Stigonema y Scytonema (Cyanobacteria), Trentepohlia y Characium (Chlorophyta), epífitas sobre una hepática y un musgo (MA 3911). Las algas fueron determinadas por Rosa María Mercado y el Dr. P. Bourrelly (Ortega, 1984). En el Herbario Nacional (MEXU) se tiene un juego de foto-exsiccata de dichas colecciones.

MÜLLER, Friedrich (siglo XIX). Explorador alsaciano (alemán) que trabajó en México en 1853 y 1854 colectando muestras de especies vegetales, posteriormente distribuidas por el señor C. Schlumberger, entre ellas algas marinas y dulceacuícolas de los phyla Cyanobacteria, Rhodophyta, Chlorophyta y Charophyta, de Orizaba, Río Blanco y Borrego, Veracruz (Hemsley, 1891; Barnhart, 1965). Sus especímenes se encuentran principalmente en el Herbario Nacional de Leiden (L) con duplicados en NY y PC; los ejemplares fueron identificados por Friedrich Traugott Kützing, ficólogo alemán y profesor en Nordhausen (Kützing, 1844, 1849a, b). A Schlumberger se le adjudica un ejemplar de Chara robbinsii Halsted (Charophyta) de 1853 de Veracruz (Ortega, 1984), el cual está depositado en NY.

OCHOA Y VILLAGÓMEZ, Ignacio (siglo XIX). Ingeniero agrónomo mexicano. En 1884 el general Carlos Pacheco, Secretario de Fomento, remitió a Alfonso Herrera (quien fungía entonces como director de la Comisión Científica Mexicana y profesor de la Escuela Preparatoria) una colección de algas de los géneros Dictyota, Padina, Sargassum, Halimeda y Nemalion de los médanos de Veracruz, obtenida por el Ing. Ignacio Ochoa y Villagómez (Pacheco, 1887). Al respecto, Ochoa y Villagómez (1887) publicó una lista de las respectivas especies de algas. Se deduce que el profesor Herrera identificó el material. Sus colecciones no han sido encontradas. 
ORCUTT, Charles Russell (1864-1929). Naturalista norteamericano y colector de plantas vasculares. Colectó algunas cianobacterias de La Grulla, Baja California y Distrito Federal y sus ejemplares se encuentran en F, PH, S.

OSORIO TAFALL, Bibiano Fernández (1902-1990). Biólogo, economista y politólogo español, exiliado en México. Profesor de la Universidad Nacional Autónoma de México y del Instituto Politécnico Nacional. Dentro de la ficología estudió las algas planctónicas del lago de Pátzcuaro, Michoacán, en 1941 y describió 12 taxa nuevos para México. Más detalles sobre su obra ficológica (1941-1944) pueden consultarse en Ortega (1984). En 1946 colectó algas marinas en Isla San Roque, Baja California Sur, las que envió a Dawson (ver esta entrada). Le fue dedicado el nombre de Myriogramme osorioi E. Y. Dawson (sinónimo de Myriogramme caespitosa E. Y. Dawson).

PALMER, Edward (1831-1911). Emigrante inglés a los Estados Unidos. Colector botánico de vastas regiones de América (McVaugh, 1956). Entre 1891 y 1896 obtuvo muestras de algas del phylum Charophyta de Durango y Baja California Sur, que se encuentran depositadas en NY y US. Dichos ejemplares fueron identificados por Timothy F. Allen de la Academia de Ciencias de Nueva York (Allen, 1880, 1888). Posteriormente un espécimen proveniente de Durango sirvió de tipo de Chara stellata Robinson (actualmente C. zeylanica Klein ex Willdenow). Le fue dedicado el nombre de C. palmeri R. D. Wood.

PATRICK, Ruth Myrtle (1907-). Prominente diatomóloga americana en Philadelphia que colectó en 1947 algunas cianobacterias del Lago de Chapultepec (Distrito Federal), Texcoco (Estado de México), Lago de Pátzcuaro (Michoacán) y en localidades de otros estados como Veracruz, Tamaulipas e Hidalgo. Sus materiales se encuentran en F y PH.

PLUMMER, Frederick Byron (1886-1947). Destacado geólogo norteamericano que incidentalmente colectó una cianobacteria en Michoacán en 1943, su ejemplar se encuentra en F.

PRINGLE, Cyrus Guernsey (1838-1911). Horticultor americano y colector que vino a México en 1885 por primera vez y desde entonces repitió su visita cada año. Al inaugurarse el Instituto Médico Nacional se le encargó colectar para esta institución. Remitió plantas a muchos museos y sus colectas ascienden a 156,000 
ejemplares de plantas vasculares (Beltrán, 1982). También recogió varios especímenes de Charophyta en Coahuila, Chihuahua, Distrito Federal (Tlalnepantla), Guanajuato, San Luis Potosí y norte de México entre 1888 y 1906. Destacan las algas Nitella opaca (C. Agardh ex Bruzelius) C. Agardh y Nitella hyalina f. formosa (T. F. Allen) R. D. Wood (como N. formosa T. F. Allen) del Valle de México en 1896; Chara haitensis Turpin de Arroyo Canoas en San Luis Potosí de 1889, debido a que fueron identificadas por los destacados ficólogos americanos Collins, Holden y Setchell (1895-1919); sus ejemplares formaron parte de la Phycoteca Boreali-Americana (PBA) resguardada en el Herbario Nacional (MEXU). Otros especimenes se encuentran en BM, MICH, NY y UC. Existen dos isotipos de México en US: Chara hydropitys var. mexicana T. F. Allen y Nitella hyalina (De Candolle) C. Agardh (como N. formosa (T. F. Allen)

PURPUS, Carl Albert (1851-1941). Farmacólogo alemán y destacado colector de plantas vasculares del occidente de Estados Unidos y México a partir de 1897. Conoció al botánico T. S. Brandegee y al ficólogo W. A. Setchell (Sousa Sánchez, 1969). Obtuvo algunos ejemplares de Charophyta de Veracruz en 1913. Sus ejemplares se encuentran en UC.

REKO, Blas Pablo (1876-1953). Médico, etnobotánico y escritor austriaco radicado en México. Colectó incidentalmente algunas algas (Chlorophyta) cerca de Pochutla, en Oaxaca, en 1941; sus ejemplares se encuentran en MEXU.

SÁMANO BISHOP, Amelia (1906-1998). Bióloga y ficóloga pionera en México, que además obtuvo otro doctorado en parasitología y embriología experimental. En 1929 fue miembro fundador del Instituto de Biología (Ortega et al., 1996) y a partir de 1947 estableció el Departamento de Embriología de la Facultad de Medicina; también fue jefa del Departamento de Biología en la Facultad de Ciencias de la Universidad Nacional Autónoma de México. En botánica estuvo dedicada al estudio de las algas de agua dulce del Valle de México. Los detalles de su obra ficológica (1932-1948) pueden consultarse en Ortega (1984). En 1932 describió una nueva especie: Chara tehuacanensis Sámano-Bishop (Charophyta, probablemente sinónimo de C. vulgaris Linnaeus) que había colectado de Tehuacán. Algunos de sus ejemplares se encuentran en MEXU.

SCHAFFNER, Wilhelm Heinrich (1830-1882). Químico farmacéutico, natural de Darmstadt (Alemania), establecido en México hacia 1856. En Kew se reci- 
bieron remesas de ejemplares de Schaffner hasta 1881 por intercesión de Asa Gray (Hemsley, 1891). Colectó organismos del phylum Charophyta. Un espécimen suyo de San Luis Potosí de 1876 sirvió para tipificar a Nitella gracilis var. asagrayana (Schaffner ex Nordstedt) R. D. Wood; se encuentra depositado en F y existe un isotipo en NY. Le fue dedicada la subespecie Chara contraria subsp. schaffneri A. Braun, descrita con base en un ejemplar de la Laguna del Valle de México de 1855 y depositado en BM; Wood e Imahori (1962) la transfieren a Chara vulgaris f. schaffneri (A. Braun) R. D. Wood.

SCHMITT, Waldo LaSalle (1887-1977). Biólogo norteamericano, miembro del personal del Instituto Smithsoniano, zoólogo de profesión y participante en la Allan Hancock Pacific Expedition a las Islas Galápagos y otras regiones del Pacífico en 1933. Estuvo también en el Presidencial Cruise de 1938 al Pacífico y México y en la 4a Expedición al Caribe (Smithsonian-Bredin Expedition) en 1960. En el Pacífico colectó macroalgas marinas de Bahía Magdalena y Cabo San Lucas (Baja California), Morro de Petatlán (Guerrero), así como en Islas Clipperton y, del lado del Caribe, en las costas de Yucatán. El material está depositado en US (incluye 2 holotipos y 1 isotipo) y otra parte en MICH. Schmitt fue el responsable principal de colectar y resguardar el material ficológico que se le enviaría al notable ficólogo W. R. Taylor (véase esta entrada) de la Universidad de Michigan (Taylor, 1939, 1972, Wynne, 1991). Le fue dedicado el nombre de Rhodymenia schmittii W. R. Taylor, entre otras especies.

SCHOTT, Arthur Carl Victor (1814-1875). Naturalista alemán. En noviembre y diciembre de 1853 Schott se encargó de hacer exploraciones topográficas y botánicas en los límites de la frontera México-Estados Unidos de América, en Boca de Río Bravo, Tamaulipas (Taylor, 1941). En este contexto también obutvo muestras de algas dulceacuícolas de Tamaulipas (1853), Coahuila (1852) y Chihuahua (1885). A su vez, entre 1864 y 1865 colectó algas marinas de la península de Yucatán en Celestún, Sisal, Progreso y Río Lagartos. Un lote de su herbario ficológico fue enviado a W. H. Harvey y posteriormente vendido al Field Museum por una modesta suma en marzo de 1897. Las algas fueron remitidas a William G. Farlow en Cambridge para su identificación. En noviembre de 1898, Farlow regresó todos los especímenes identificados al Field Museum (F); sin embargo éstos no se dieron a conocer sino hasta el siglo XX. Taylor (1941) registró 89 ejemplares marinos para México de Cyanobacteria, Rhodophyta, Phaeophyceae y Chlorophyta. Murray (1891), del Museo Británico (BM), descubrió que un ejemplar colectado por Schott era una nueva especie (Caulerpa phyllophlaston Murray) de Progreso, Yucatán. Weber van Bosse 
(1898) posteriormente la consideró como Caulerpa paspaloides f. phyllophlaston (Murray) Weber van Bosse. También uno de sus ejemplares de Boca del Río Bravo resultó ser sintipo de Chaetomorpha brachygona Harvey. Le fue dedicado el nombre de Protokuetzingia schottii W. R. Taylor (sinónimo de Halopithys schottii (W. R. Taylor) L. E. Phillips \& De Clerck).

SOCOLOV, Dimitri (Sokoloff, Demetrio) (1891-1973). Doctor en ciencias naturales de la Universidad de Moscú, oriundo de esas tierras y posteriormente radicado en México. Al poco tiempo de fundarse el Instituto de Biología ingresó como investigador del Laboratorio de Zoología e Hidrobiología. Estudió principalmente algas flageladas, de las que describió algunas especies nuevas del Valle de México (Caballero y Caballero, 1974). Más detalles de su obra ficológica (1930-1937) pueden consultarse en Ortega (1984).

STANSCH, Carlos (Karl) (siglo XIX). Personaje de origen alemán, radicado en México, donde trabajó como inspector de la Dirección Forestal de Caza y Pesca, perteneciente a la Secretaría de Agricultura y Fomento, creada en 1917. Colectó material ficológico de los phyla Cyanobacteria, Rhodophyta y Chlorophyta para la Dirección de Estudios Biológicos (antecedente inmediato del Instituto de Biología, de la Universidad Nacional Autónoma de México), del Lago de Chapultepec, Lago de Xochimilco y Tlalpan (Distrito Federal, 1923); de Baja California (1911); de Islas Tres Marías (Nayarit, 1927), así como de Escuinapa y Teacapan (Sinaloa, 19241927). Su material se encuentra en MEXU. Se deduce que él mismo identificó sus ejemplares. Le fue dedicado el nombre de una especie de crustáceo: Gonodactylus stanschi por W. L. Schmitt (1940), véase esta entrada.

STENIK, K. Colector acerca del cual no se tienen mayores noticias, pues únicamente se sabe que obtuvo un ejemplar de Charophyta en Hidalgo en 1924, mismo que se encuentra depositado en MEXU.

TAYLOR, William Randolph (1895-1990). Notable ficólogo norteamericano. Participó en la Expedición del Capitán Allan Hancock a bordo del Velero III en 1934 y 1939, desde el Pacífico mexicano hasta Islas Galápagos. Recolectó macroalgas marinas de Islas Marías, Nayarit; Islas Revillagigedo, Colima; Bahía Tenacatita, Jalisco; Bahía de Petatlán, Guerrero; Isla Tangola-Tangola y Bahía de Chacahua, Oaxaca (Taylor, 1945). Identificó muchos ejemplares de la Smithsonian - Bredin Expedition de 1960 a Yucatán, recolectadas por W. L. Schmitt (véase esta entrada). Sus ejemplares se 
encuentran en UC y otros en MICH. Fue pionero en los trabajos monográficos, como el de 1960 sobre las algas tropicales y subtropicales de la costa oriental de América. Su amplia bibliografía puede consultarse en Wynne (1991). Le dedicaron el nombre de Amphiroa taylorii E. Y. Dawson, entre muchos otros.

THOMAS, John H. Integrante de la expedición al sur de Baja California de la Academia de Ciencias de California. Colectó una Charophyta en el arroyo San Mateo en Baja California en 1959 cuyo ejemplar se encuentra en MEXU.

VIVES, Gastón J. (1859-1939 aprox.). Colector mexicano de algas marinas de los phyla Ochrophyta y Chlorophyta en Bahía de la Paz y Bahía San Felipe en el Golfo de California en 1911. Los ejemplares se encuentran en el Herbario Nacional (MEXU). La identificación de sus especímenes fue dada a conocer por M. A. Howe (1911), el cual le dedica los nombres de dos especies de algas: Dictyota vivesii Howe (sinónimo de D. dichotoma (Hudson) Lamouroux) y Gracilaria vivesii Howe.

\section{CONSIDERACIONES FINALES}

En el período de estudio suman 51 colectores de algas cuyos ejemplares se encuentran depositados en 16 herbarios de Europa, América y Australia. En el Herbario Nacional (MEXU) se localizan importantes materiales de Arsène, Pringle, Stansch y Vives. Se tiene referencia que algunos mexicanos como Ochoa y Villagómez, Osorio Tafall y Socolov también recolectaron algas en el territorio del país (Ortega, 1984; Ortega et al., 2001); sin embargo los ejemplares no quedaron depositados en herbarios institucionales.

Los colectores de algas de México enriquecieron el conocimiento de la flora ficológica de México en el período 1787-1954 en los phyla: Cyanobacteria, Rhodophyta, Ochrophyta (incluye Phaeophyceae y Xanthophyceae), Bacillariophyta, Chlorophyta y Charophyta. Sus materiales provienen de 26 estados: Baja California, Baja California Sur, Campeche, Chiapas, Chihuahua, Coahuila, Colima, Distrito Federal, Durango, Estado de México, Guanajuato, Hidalgo, Jalisco, Michoacán, Morelos, Nayarit, Nuevo León, Oaxaca, Puebla, Quintana Roo, San Luis Potosí, Sinaloa, Sonora, Tamaulipas, Yucatán, Zacatecas, así como de otras localidades como Banco de Campeche (Sonda de Campeche), Golfo de México, Laguna del Valle de México, litoral Océano Pacífico, México, norte de México y Pacífico mexicano. Los grupos mayoritarios fueron las Bacillariophyta y Rhodophyta. Con base en los acervos acu- 
mulados de esta manera, en el período estudiado se describieron numerosas nuevas especies para la ciencia (477 aprox.). Un total de 24 botánicos y ficólogos estudiaron el material mexicano en el lapso de los 167 años. La mayoría fueron norteamericanos (9), le siguieron los suecos (5), alemanes (4), británicos (2) y uno francés; sin embargo hay que destacar el papel de los mexicanos (5), como Sámano Bishop, pionera de la ficología en México.

\section{AGRADECIMIENTOS}

Se agradece a los curadores del Museo Botánico de Copenhague, Museo Sueco de Historia Natural de Estocolmo, Museo Botánico de Lund, Riksherbarium en Leiden, Museo de Historia Natural de Londres, Museo Nacional de Historia Natural de París y Jardín Botánico de Madrid, por las facilidades otorgadas para la consulta de los herbarios. Especialmente merecen reconocimiento los doctores P. C. Silva de la Universidad de California en Berkeley, el Dr. D. Lazarus del Museo de Historia Natural de Berlín, la Dra. C. Gallagher del National Herbarium of Victoria (MEL), Royal Botanic Gardens Melbourne, la Dra. Paloma Blanco del Jardín Botánico de Madrid y el Dr. M. J. Wynne de la Universidad de Michigan (Ann Arbor), por su información sobre la colecciones de algas de México. Deseo agradecer igualmente al personal de la biblioteca del Instituto de Biología y al Lic. Amando Butanda por su ayuda eficiente en la búsqueda bibliográfica, al Dr. Jerzy Rzedowski por la lectura detallada y crítica del trabajo, la cual mejoró grandemente su contenido, así como también a los revisores anónimos.

\section{LITERATURA CITADA}

Abbott, I. A. 1966. Elmer Yale Dawson (1918-1966). J. Phycol. 2: 129-132.

Agardh, C. A. 1822. Algae. In: Kunth, C. S. Synopsis plantarum, quas, in itinere ad plagam aequinoctialem orbis novi, collegerunt Al. de Humboldt et Am. Bonpland. Vol. 1. Parisiis (París). pp. 1-6.

Agardh, J. G. 1847. Nya Alger från Mexico. Öfvers. Förh. Kongl. Svenska Vetensk.-Akad. 4(1): 5-17.

Agardh, J. G. 1848. Neue Algen aus Mexico. Flora 31(25): 404-416.

Allen, T. F. 1880. The Characeae of America. Boston. Part 1: 1-8, Part 2: 9-14.

Allen, T. F. 1888. The Characeae of America. Part I. Introduction, morphology and classification. Nueva York. pp. 1-64. 
Anónimo. 1986. Diccionario Porrúa de historia, biografía y geografía de México. Editorial Porrúa. México, D.F. 1126 pp.

Barnhart, J. H. 1965. Biographical notes upon botanists. Vol. 2. Hall \& Co. Boston. 549 pp. Beck, H. 1971. Alexander von Humboldt. Fondo de Cultura Económica. México, D.F. 491 pp. Beltrán, E. 1982. Contribución de México a la biología, pasado, presente y futuro. Compañía Editorial Continental, CECSA. México, D.F. 121 pp.

Bonpland, A., A. de Humboldt y C. S. Kunth. 1825. Nova genera et species plantarum. Tomus septimus, Lutetiae Parisiorum (París), Apud Gide filium, Bibliopolam. 399 pp.

Caballero y Caballero, E. 1974. Dimitri Socolov 1891-1973. An. Inst. Biol. Univ. Nal. Autón. México, ser. Zoología 45(1): 151-152.

Campos-Ríos, M. G. y F. Chiang Cabrera. 2006. Una revisión nomenclatural de los tipos de plantas de la Península de Yucatán (México). Polibotánica 22: 89-149.

Chaudhri, M. N., I. H. Vegter y C. M. De Wal. 1972. Index Herbariorum. Part II (3). Collectors (I-L). Regn. Veget. 86: 297-473.

Christensen, C. 1924-1926. Den danske botaniks historie med tilhorende bibliografi. 3 vols. H. Hagerups Forlag. København (Copenhague).

Collins, F. S., I. Holden y W. A. Setchell. 1895-1919. Phycoteca Boreali-Americana. A collection of dried specimens of the algae of North America. Malden, Massachusetts. Fascicle E.

Dawson, E. Y. 1944. The marine algae of the Gulf of California. Allan Hancock Pac. Exp. 3(10): 89-453.

Dawson, E. Y. 1953. Resumen de las investigaciones recientes sobre algas marinas de la costa pacífica de México, con una sinopsis de la literatura, sinonimia y distribución de las especies descritas. Rev. Soc. Mex. Hist. Nat. 13: 97-197.

Dawson, E. Y. 1966. New records of marine algae from the Gulf of California. J. Ariz. Acad. Sci. 4(2): 55-66.

Ducker, S. C. 1981. Australian phycology: the German influence. In: Carr D. J. y S. G. M. Carr (eds.). People and plants in Australia. Academic Press. Sydney. pp. 116-138.

Ehrenberg, C. G. 1841 (1843). Verbreitung und Einflufs des mikroscopischen Lebens in Südund Nord-Amerika. Abh. Königl. Akad. Wiss. Belin, Phys. K1. 1841: 291-445.

Ehrenberg, C. G. 1854. Mikrogeologie. Verlag von Leopold Voss. Leipzig. XXVIII + 374 pp.

Ehrenberg, C. G. 1874. De la toba fitolitaria del Valle de Toluca. La Naturaleza (México) 1a ser., 3: 118-132.

Fisher, W. K. 1905. In memorian: Walter E. Bryant. Condor 7(5): 129-131.

Forest, H. 1983. Francis Drouet. March 1, 1907 - December 5, 1982. Bull. Torrey Bot. Club. 110: 231-238.

Gardner, N. L. 1927. New Rhodophyceae from the Pacific coast of North America. III. Univ. Calif. Publ. Bot. 13(16): 333-368.

Garth, J. S. 1967. E. Yale Dawson 1918-1966. Bull. South. Calif. Acad. Sci. 66: 149-160.

Harriot, P. 1895. Algues du Golfe de Californie, recueillies par M. Diguet. J. Bot. (Morot) 9: $167-170$.

Hawkes, 2005. M. W. In search of cacti and seaweeds on desert shores: E. Yale Dawson (1918-1966), botanist. Haseltonia 11: 126-137. 
Hemsley, W. B. 1891. Bosquejo de la historia de la exploración botánica de México. La Naturaleza (México) 1a ser. 1: 1-15.

Herrera, T., M. M. Ortega, J. L. Godínez y A. Butanda. 1998. Breve historia de la botánica en México. Fondo de Cultura Económica. México, D.F. 168 pp.

Hirn, E. K. 1900. Monographie und Iconographie der Oedogoniaceen. Acta Soc. Sci. Fenn. 27: IV $+394 \mathrm{pp}$.

Holmgren, K. H., N. H. Holmgren y L. C. Barnett. 1990. Index Herbariorum. Part 1: The herbaria of the world. 8a ed. New York Botanical Garden. Nueva York. 693 pp.

Holmgren, P. K. y N. H. Holmgren. 1998 (continuously updated). Index Herbariorum: A global directory of public herbaria and associated staff. New York Botanical Garden's Virtual Herbarium. http://sweetgum.nybg.org/ih/

Howe, M. A. 1911. Phycological studies-V. Some marine algae of Lower California, Mexico. Bull. Torrey Bot. Club 38: 489-514.

Kützing, F. T. 1844. Die kieselschaligen Bacillarien oder Diatomeen. W. Köhne. Nordhausen. pp.1-152.

Kützing, F. T. 1849a. Species algarum. F. A. Brockhaus. Lipsiae (Leipzig). vi + 922 pp.

Kützing, F. T. 1849b. Tabulae phycologicae oder Abbildungen der Tange. Vol. I, fasc. 6-10. Nordhausen. pp. 37-54.

Lanjouw, J. y F. A. Stafleu. 1954. Index Herbariorum. Part. II. Collectors (A-D). Regn. Veget. 2: 5-174.

Lanjouw, J. y F. A Stafleu. 1957. Index Herbariorum. Part. II (2). Collectors (E-H). Regn. Veget. 9: 175-295.

Lazarus, D. B. y R. Jahn. 1998. Using the Ehrenberg collection. Diatom Res. 13(2): 273-291.

Liebmann, F.M. 1846. Beretning om Amerikas Vandplanter af Algernes Classe. Overs. Kongel. Danske Vidensk. Selsk. Forh. Medlemmers Arbeider 1846: 72-77.

Liebmann, F. M. 1869. Chênes de l'Amérique tropicale. Leipzig. 29 pp. (Trabajo póstumo con adiciones de A. S. Oersted, incluye biografía y biliografía de Liebmann).

McVaugh, R. 1956. Eward Palmer. Plant explorer of the American West. University of Oklahoma Press. Norman. 430 pp.

McVaugh, R. 1987. Itinerary and gazetteer. In: Liebmann's Mexican ferns. Contr. New York Bot. Gard. 19: 5-38.

Murray, G. 1891. On new species of Caulerpa, with observations on the position of the genus. Trans. Linn. Soc. London, Bot. Ser. 2, 3: 207-213.

Ochoa y Villagómez, I. 1887. Vegetación espontánea y repoblación de los médanos de la zona litoral de Veracruz. Informe presentado a la Secretaría de Fomento. Memorias de la Secretaría de Fomento, México (1883-1885) 3: 641-655.

Ortega, M. M. 1984. Catálogo de algas continentales recientes de México. Instituto de Biología, Universidad Nacional Autónoma de México. México, D.F. 566 pp.

Ortega, M. M. 1987. Doce años de ficología en México (971-1983). In: Gómez Aguirre. S. y V. Arenas Fuentes (eds.). Contribuciones en hidrobiología. Memoria de la Reunión Alejandro Villalobos (24 al 26 de octubre de 1983). Universidad Nacional Autónoma de México. México, D.F. pp. 155-186.

Ortega, M. M. y J. L. Godínez. 1994. Perspectivas de la ficología en México. Bol. Soc. Bot. México 55: 115-122. 
Ortega, M. M., J. L. Godínez y G. Vilaclara. 1996. Relación histórica de los antecedentes y origen del Instituto de Biología. Instituto de Biología, Universidad Nacional Autónoma de México. México, D.F. 98 pp.

Ortega, M. M., J. L. Godínez y G. Garduño Solórzano. 2001. Catálogo de algas bénticas de las costas mexicanas del Golfo de México y Mar Caribe. Cuadernos del Instituto de Biología, Universidad Nacional Autónoma de México 34: 1-594.

Pacheco, C. 1887. Carta dirigida por C. Pacheco a A. Herrera en la que comunica los objetos coleccionados por el Ing. Ignacio Ochoa Villagómez en su expedición a la zona litoral de Veracruz. In: Anexo 43, Comisión Científica. Memorias de la Secretaría de Fomento, México (1883-1885) 4: 447-448.

Papenfuss, G. F. 1976. Landmarks in Pacific North American marine phycology. In: Abbott I. A. y G. J. Hollenberg (eds.). Marine algae of California. Stanford University Press. Stanford, CA. pp. 21-46.

Pedroche, F. F. y A. Sentíes Granados. 2003. Exploración ficológica en el litoral del Océano Pacífico. In: Robledo Ramírez, D., J. L. Godínez Ortega y Y. Freile Pelegrín (eds.). Contribuciones ficológicas de México. Sociedad Ficológica de México, A.C. Mérida, Yucatán. pp. 5-11.

Pedroche, F. F., P. C. Silva, L. E. Aguilar Rosas, K. M. Dreckmann y R. Aguilar Rosas. 2003. Macroalgas marinas béntica del Pacífico. Referencias bibliográficas selectas (1847-2002). In: Robledo Ramírez, D., J. L. Godínez Ortega y Y. Freile Pelegrín (eds.). Contribuciones ficológicas de México. Sociedad Ficológica de México, A.C. Mérida, Yucatán, México. pp. 97-126.

Pedroche, F. F., P. C. Silva, L. E. Aguilar Rosas, K. M. Dreckmann y R. Aguilar Rosas. 2005. Catálogo de las algas marinas bentónicas del Pacífico de México I. Chlorophycota. Universidad Autónoma de Baja California, Universidad Autónoma Metropolitana Unidad Iztapalapa y University of California, Campus Berkeley. Mexicali, Baja California, México. 135 pp.

Purata Velarde, S. E. 1981. Las colecciones botánicas del hermano G. Arsène. Tesis profesional. Facultad de Ciencias, Universidad Nacional Autónoma de México. México, D.F. 96 pp.

Schmitt, W. L. 1940. The stomatopods of the west coast of America. Allan Hancock Pac. Exped. 5(4): 129-225.

Setchell, W. A. 1937. Nathaniel Lyon Gardner (1864-1937). Madroño 4: 126-128.

Setchell, W. A. y N. L. Gardner. 1924. Expedition of the California Academy of Sciences to the Gulf of California in 1921. The marine algae. Proc. Calif. Acad. Sci. ser. 4, 12: 695-949.

Setchell, W. A. y N. L. Gardner. 1930. Marine algae of the Revillagigedo Islands Expedition in 1925. Proc. Calif. Acad. Sci. ser.4, 19: 109-215

Setchell, W. A. y N. L. Gardner. 1937. The Templeton Crocker Expedition of the California Academy of Sciences, 1932. No. 31. A preliminary report on the algae. Proc. Calif. Acad. Sci. ser. 4, 22: 65-98.

Silva, P. C. 1967. E. Yale Dawson (1918-1966). Phycologia 6(4): 218-236.

Sousa Sánchez, M. 1969. Las colecciones botánicas de C. A. Purpus en México: período 1898-1925. Univ. Calif. Pub. Bot. 51: 1-36. 
Stafleu, F. A. y R. S. Cowan. 1976. Taxonomic literature. A selective guide to botanical publications and collections with dates, comentaries and types. Vol. I: A-G. 2a ed. Bohn, Scheltema \& Holkema. Utrecht. 1136 pp.

Stafleu, F. A. y R. S. Cowan. 1979. Taxonomic literature. A selective guide to botanical publications and collections with dates, comentaries and types. Vol. II: H-Le. 2a ed. Bohn, Scheltema \& Holkema. Utrecht. 991 pp.

Stafleu, F. A. y R. S. Cowan. 1981. Taxonomic literature. A selective guide to botanical publications and collections with dates, comentaries and types. Vol. III: Lh-O. 2a ed. Bohn, Scheltema \& Holkema. Utrecht. 980 pp.

Stafleu, F. A. y R. S. Cowan. 1983. Taxonomic literature. A selective guide to botanical publications and collections with dates, comentaries and types. Vol. IV: P-Sak. 2a ed. Bohn, Scheltema \& Holkema. Utrecht. 1214 pp.

Stafleu, F. A. y R. S. Cowan. 1985. Taxonomic literature. A selective guide to botanical publications and collections with dates, comentaries and types. Vol. V: Sal-Ste. 2a ed. Bohn, Scheltema \& Holkema. Utrecht. 1066 pp.

Stafleu, F. A. y R. S. Cowan. 1986. Taxonomic literature. A selective guide to botanical publications and collections with dates, comentaries and types. Vol. VI: Sti-Vuy. 2a ed. Bohn, Scheltema \& Holkema. Utrecht. 926 pp.

Taylor, W. R. 1939. Algae collected on the Presidental Cruise of 1938. Smithsonian Misc. Collect. 98: 1-18

Taylor, W. R., 1941. Tropical marine algae of the Arthur Schott Herbarium. Publ. Field Mus. Nat. Hist., Bot. Ser. 20(4): 87-104.

Taylor, W.R. 1945. Pacific marine algae of the Allan Hancock Expeditions to the Galapagos Islands. Allan Hancock Pac. Exped. 12: iv +528.

Taylor, W. R. 1972. Marine algae of the Smithsonian-Bredin expedition to Yucatan-1960. Bull. Mar. Sci. 22(1): 34-44.

Vegter, I. H. 1976. Index Herbariorum. Part II (4) Collectors (M). Regn. Veget. 93: 475576.

Vegter, I. H. 1983. Index Herbariorum. Part II (5) Collectors (N-R). Regn. Veget. 109: 577803.

Weber van Bosse, A. A. 1898. Monographie des Caulerpes. Ann. Jard. Bot. Buitenzorg 15: 243-401.

Wood, R. D. y K. Imahori. 1962. Monograph of the Characeae. Verlag von J. Cramer. Weinheim. 904 pp.

Wynne, M. J. 1991. Prof. Dr. William Randolph Taylor. Bot. Mar. 34: 63-67.

Recibido en mayo 2008. 
